\title{
School-based surveillance of acute infectious disease in children: a systematic review
}

\author{
A. L. Donaldson ${ }^{1,2,3^{*}}$ (D, J. L. Hardstaff ${ }^{1,2}$, J. P. Harris ${ }^{1,2}$, R. Vivancos ${ }^{2,3}$ and S. J. O'Brien ${ }^{1,2}$
}

\begin{abstract}
Background: Syndromic surveillance systems are an essential component of public health surveillance and can provide timely detection of infectious disease cases and outbreaks. Whilst surveillance systems are generally embedded within healthcare, there is increasing interest in novel data sources for monitoring trends in illness, such as over-the-counter purchases, internet-based health searches and worker absenteeism. This systematic review considers the utility of school attendance registers in the surveillance of infectious disease outbreaks and occurrences amongst children.
\end{abstract}

Methods: We searched eight databases using key words related to school absence, infectious disease and syndromic surveillance. Studies were limited to those published after 1st January 1995. Studies based in nursery schools or higher education settings were excluded. Article screening was undertaken by two independent reviewers using agreed eligibility criteria. Data extraction was performed using a standardised data extraction form. Outcomes included estimates of absenteeism, correlation with existing surveillance systems and associated lead or lag times.

Results: Fifteen studies met the inclusion criteria, all of which were concerned with the surveillance of influenza. The specificity of absence data varied between all-cause absence, illness absence and syndrome-specific absence. Systems differed in terms of the frequency of data submissions from schools and the level of aggregation of the data. Baseline rates of illness absence varied between 2.3-3.7\%, with peak absences ranging between 4.1-9.8\%. Syndrome-specific absenteeism had the strongest correlation with other surveillance systems $(r=0.92)$, with illness absenteeism generating mixed results and all-cause absenteeism performing the least well. A similar pattern of results emerged in terms of lead and lag times, with influenza-like illness (ILI)-specific absence providing a 1-2 week lead time, compared to lag times reported for all-cause absence data and inconsistent results for illness absence data.

Conclusion: Syndrome-specific school absences have potential utility in the syndromic surveillance of influenza, demonstrating good correlation with healthcare surveillance data and a lead time of 1-2 weeks ahead of existing surveillance measures. Further research should consider the utility of school attendance registers for conditions other than influenza, to broaden our understanding of the potential application of this data for infectious disease surveillance in children.

\footnotetext{
* Correspondence: A.Donaldson2@liverpool.ac.uk

${ }^{1} \mathrm{NIHR}$ Health Protection Research Unit in Gastrointestinal Infections,

University of Liverpool, Liverpool, UK

${ }^{2}$ Institute of Population Health Sciences, University of Liverpool, Liverpool, UK

Full list of author information is available at the end of the article
}

(c) The Author(s). 2021 Open Access This article is licensed under a Creative Commons Attribution 4.0 International License, which permits use, sharing, adaptation, distribution and reproduction in any medium or format, as long as you give appropriate credit to the original author(s) and the source, provide a link to the Creative Commons licence, and indicate if changes were made. The images or other third party material in this article are included in the article's Creative Commons licence, unless indicated otherwise in a credit line to the material. If material is not included in the article's Creative Commons licence and your intended use is not permitted by statutory regulation or exceeds the permitted use, you will need to obtain permission directly from the copyright holder. To view a copy of this licence, visit http://creativecommons.org/licenses/by/4.0/ The Creative Commons Public Domain Dedication waiver (http://creativecommons.org/publicdomain/zero/1.0/) applies to the data made available in this article, unless otherwise stated in a credit line to the data. 
Systematic review registration: PROSPERO 2019 CRD42019119737.

Keywords: School absence, School attendance registers, Children, Syndromic surveillance, Infectious disease, Outbreaks

\section{Background}

Public Health surveillance is the "continuous, systematic collection, analysis and interpretation of health-related data needed for the planning, implementation, and evaluation of public health practice." [1] For infectious disease, timely surveillance systems are fundamental to providing early detection of cases and outbreaks, allowing measures to be put in place to protect others and reduce transmission [2]. Historically, public health surveillance was disease-specific, relying on clinical diagnoses and laboratory reports [3]. However, such surveillance systems can be subject to significant delays and over recent years there has been increasing recognition of the value of syndromic surveillance in providing more timely detection of infectious illness [4-7].

Syndromic surveillance can be based on either the identification of clinical syndromes that are indicative of a given disease, or the clustering of non-specific symptoms and changes in patterns of health behaviours which could indicate an outbreak or unusual event [8]. Syndromic surveillance systems have been developed using multiple sources of data, many of which are embedded within healthcare, such as emergency department attendances, ambulance dispatches or calls to remote telehealth services [9-14]. However, there is increasing interest in the use of novel sources of data, such as over-the-counter purchases, internet-based health searches and worker absenteeism, which have been found to correlate well with traditional surveillance measures [11, 15-21].

School attendance registers offer a novel dataset which could be used to provide more timely information regarding infectious disease and outbreaks amongst children [22]. Children are commonly affected by gastrointestinal illness and respiratory illness, both of which are key causes of illness absence from school [23-26]. Children are recognised as important transmitters of infection, [27-30] and schools are principal settings in the spread of infections between children [29, 31]. Close household contact with parents and grandparents facilitates the spread of illness from schools into the wider community [32, 33]. School absence data could support the early identification of outbreaks within schools, enabling timely intervention to reduce the transmission of infections both within and outside of the school setting. Furthermore, as school absence may occur from the first day of illness, this novel dataset has the potential to offer more timely data than healthcare-based surveillance.
There is evidence that children may be the first affected by seasonal and pandemic illnesses, [34-36] and by enhancing the detection of disease in children such data could provide early warning of infections before they start circulating in the wider community.

This systematic review considers the utility of school attendance registers in the surveillance of infectious disease outbreaks and occurrences amongst children. The value of a school-based surveillance system will be considered in terms of its correlation and lead time compared to traditional surveillance measures. A secondary objective of this review is to describe the burden of illness absenteeism and outbreaks in school-aged children.

\section{Methods \\ Protocol and registration}

The systematic review protocol was registered on PROSPERO in January 2019 (PROSPERO 2019 CRD42019119737) [37]. The protocol and article follow the PRISMA checklist for the reporting of systematic reviews.

\section{Eligibility criteria}

The population of interest for this review was children aged between 4 and 18 years, attending school. Only studies published on or after 1st January 1995 and available in English were included. As this review considers what school attendance data adds to existing health surveillance systems, studies were limited to those from OECD countries, [38] which are likely to have established health surveillance systems in place for comparison. No limitation was put on school type, but studies based in nursery schools or higher education settings were excluded, as these settings are not components of compulsory education and may be subject to different requirements for attendance and absence reporting. Review papers, editorials, book chapters, conference abstracts or proceedings, randomised controlled trails and case reports were also excluded. Following the full text review of articles, qualitative studies and statistical papers exploring novel mathematical techniques to modelling disease surveillance data were added to the excluded study types.

\section{Information sources}

The following electronic databases were searched: Medline, Web of Science, Pubmed, Scopus, Science 
Direct, Biosis Previews, Open Grey and Proquest dissertations and theses. The searches were conducted on 23 October 2018.

\section{Search terms}

The following search terms were used: ((population surveillance/ or public health surveillance/ or sentinel surveillance/ or surveillance .mp.) OR (syndromic surveillance.mp.) OR (attend*.mp.) OR (absenteeism/ or absen".mp.) OR (registers.mp.)) AND ((school.mp. or Schools/) OR (school aged children.mp.) OR (school children.mp.)) AND ((Infectious disease.mp. or Communicable Diseases/) OR (Outbreaks.mp. or Disease Outbreaks/) OR (epidemics.mp. or EPIDEMICS/) OR (pandemics.mp. or PANDEMICS/) OR (bugs.mp.)). The search terms were piloted before use and combined using Boolean operators. The search terms were developed for use in MEDLINE. Where possible, the same terms were used in each database, but some adaptation or simplification was required to meet the search requirements of different databases (Additional file 1). The terms were searched for within the title and abstracts of papers and, where possible, the keywords.

\section{Study selection}

References from each database were imported into Mendeley reference manager. Each reference list was first deduplicated, before combining all references and conducting a further removal of duplicate references. Additional duplicates were removed by manual searching. Two independent reviewers (AD and JLH) then undertook screening of the article titles and abstracts, applying the agreed exclusion and inclusion criteria. Any discrepancies between the reviewers were discussed and consensus reached. Articles meeting the screening criteria underwent a full text review. This was conducted by two reviewers (AD and JPH) using agreed eligibility criteria. Consensus was reached between the reviewers about the final articles for inclusion. Reference lists of the included articles were searched to identify any additional relevant studies not identified as part of the original search strategy. Papers identified in this way underwent the same screening and full text review outlined above

\section{Data collection process and data items}

Data extraction was performed using a standardised data extraction form (Additional file 2). Where available, the following data items were extracted; year of publication, country, prospective or retrospective study, age group, school type, sample size, time period of data collection, organism/syndrome, purpose of surveillance (case ascertainment or outbreak detection), case or outbreak definition, primary outcome measure, description of surveillance system (including the specificity, timeliness and spatial-temporal level of data collected), comparator surveillance systems, absenteeism rates with $95 \%$ confidence intervals, correlation measures with $p$-values, and lead or lag times compared to other surveillance measures.

\section{Summary measures}

The summary measures were descriptive of the school surveillance systems and the methods used within each study. Outcomes included estimates of absenteeism, correlation measures and lead/lag times. Due to a high level of heterogeneity, estimates could not be pooled between studies.

\section{Synthesis of results}

A narrative synthesis approach was adopted, comparing and contrasting the school-based surveillance systems in terms of their design, purpose, population, and performance against existing health surveillance systems.

\section{Results \\ Study selection}

The initial searches identified 5022 references, which reduced to 2684 once duplicates were removed. After screening the abstracts, 33 studies met the eligibility criteria for full text review. Of these, 14 were included in the systematic review. Nine additional studies were identified through searching the references of the papers for inclusion. Following abstract screening, three underwent full text review, one of which was subsequently included in the systematic review, giving a total of 15 studies (Fig. 1).

\section{Characteristics of included studies}

All of the studies identified were concerned with the surveillance of influenza and over half were related to pandemic influenza. This is reflected in a peak of studies published between 2010 and 2013, following the H1N1 pandemic in 2009 (Fig. 2). The greatest number of studies identified originated from the USA $(n=6)$, with multiple studies also reported from the UK $(n=4)$ and Canada $(n=2)$.

A summary of the included studies is outlined in Table 1 . Over half (9/15 studies) collected prospective data, the majority of which were during the H1N1 pandemic. Sample size varied from six schools to over 3000 schools. Most studies included data on all school age groups, ranging from 3 to 18 years of age.

\section{Description of methods used for school-based surveillance}

The three most common forms of absence data were allcause absenteeism, [39-42] illness absenteeism, [21, 43-48] and syndrome-specific absenteeism, which in these studies corresponded to influenza-like-illness (ILI) 


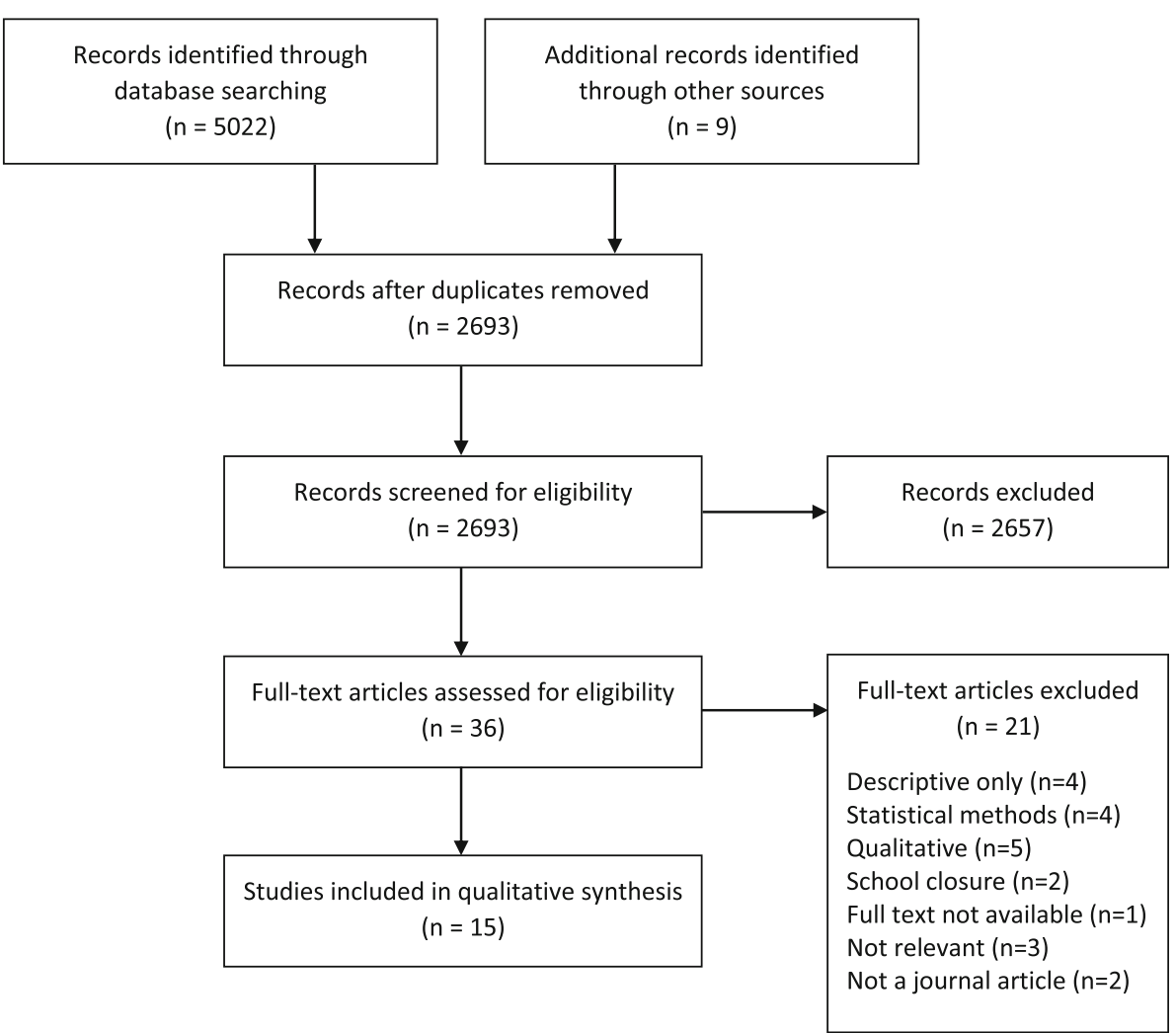

Fig. 1 Flow diagram of study selection

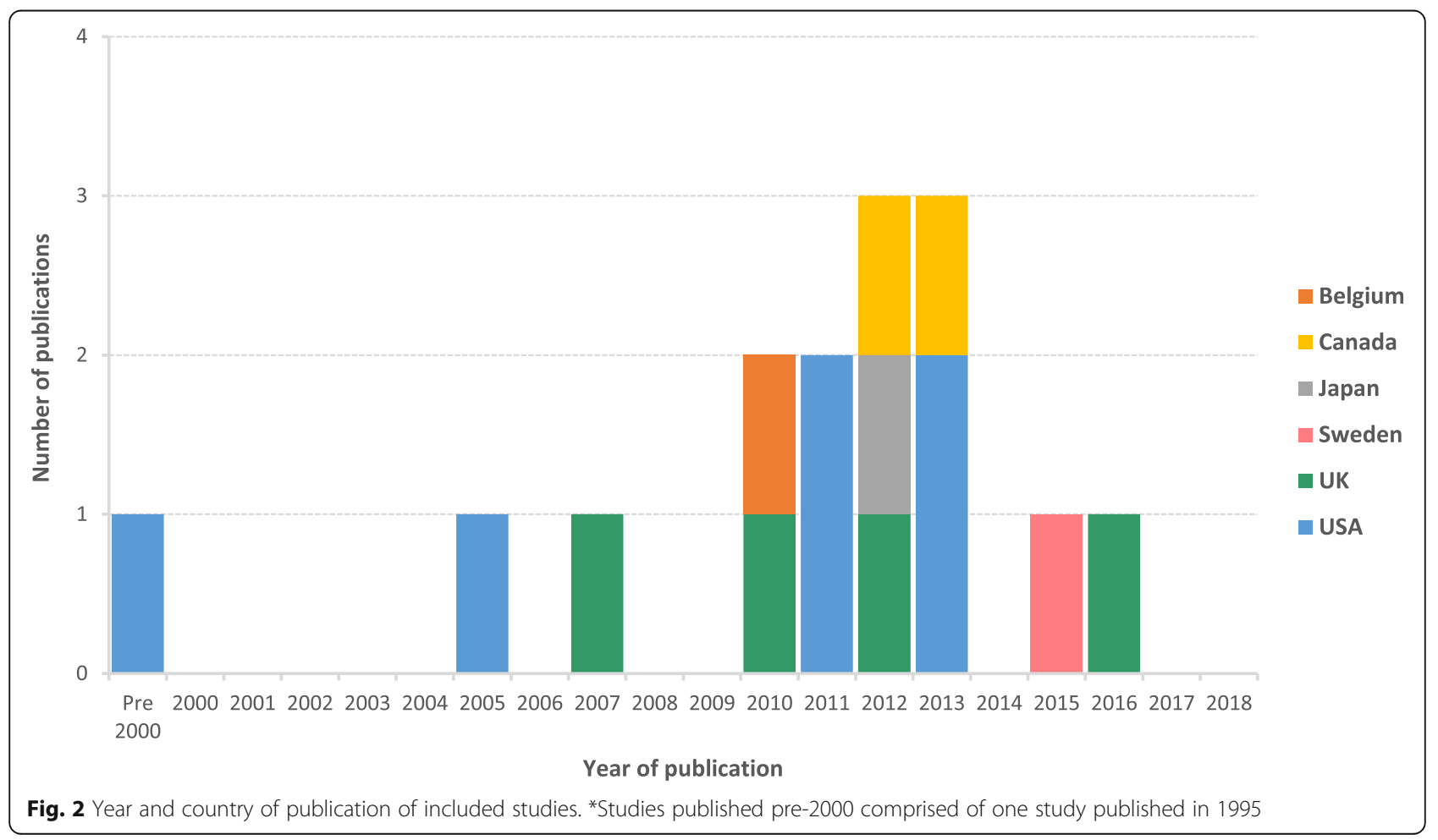


Table 1 Description of included studies

\begin{tabular}{|c|c|c|c|c|c|c|c|c|}
\hline Author & $\begin{array}{l}\text { Year of } \\
\text { Publication }\end{array}$ & Country & $\begin{array}{l}\text { Organism/ } \\
\text { syndrome }\end{array}$ & $\begin{array}{l}\text { Prospective or } \\
\text { retrospective }\end{array}$ & $\begin{array}{l}\text { School } \\
\text { ages }\end{array}$ & Sample size & $\begin{array}{l}\text { Specificity of } \\
\text { absence }\end{array}$ & $\begin{array}{l}\text { Frequency of } \\
\text { data submissions }\end{array}$ \\
\hline Aldridge et al [51] & 2016 & UK & $\begin{array}{l}\text { Seasonal } \\
\text { influenza }\end{array}$ & Prospective & $11-16 y r s$ & 27 schools & Medical $^{a}$ & Weekly \\
\hline Besculides et al [39] & 2005 & USA & $\begin{array}{l}\text { Seasonal } \\
\text { influenza }\end{array}$ & Retrospective & $5-18 \mathrm{yrs}$ & 1160 schools & All cause & Daily \\
\hline Bollaerts et al. [21] & 2010 & Belgium & $\begin{array}{l}\text { Pandemic } \\
\text { influenza }\end{array}$ & Prospective & $3-18$ yrs & $\begin{array}{l}\text { } 1 \text { million } \\
\text { pupils }\end{array}$ & Illness & Weekly \\
\hline Chu et al [52] & 2013 & Canada & $\begin{array}{l}\text { Pandemic } \\
\text { influenza }\end{array}$ & Retrospective & 4-14 yrs & $8 \mathrm{PHUs}^{\mathrm{b}}$ & Variable & Not specified \\
\hline Crawford et al [40] & 2011 & USA & $\begin{array}{l}\text { Pandemic } \\
\text { influenza }\end{array}$ & Prospective & $5-12$ yrs & 80 schools & All cause & Daily \\
\hline Kara et al [43] & 2012 & UK & $\begin{array}{l}\text { Pandemic } \\
\text { influenza }\end{array}$ & Retrospective & $4-18 y r s$ & 373 schools & Illness & Weekly \\
\hline Kightlinger et al [44] & 2013 & USA & $\begin{array}{l}\text { Pandemic } \\
\text { influenza }\end{array}$ & Prospective & $5-18$ yrs & 187 schools & Illness & Weekly \\
\hline Kom Mogto et al [49] & 2012 & Canada & $\begin{array}{l}\text { Pandemic } \\
\text { influenza }\end{array}$ & Prospective & $6-17 y r s$ & 3432 schools & $\begin{array}{l}\text { Syndrome- } \\
\text { specific }\end{array}$ & Daily \\
\hline Lenaway et al [45] & 1995 & USA & $\begin{array}{l}\text { Seasonal } \\
\text { influenza }\end{array}$ & Prospective & $5-18$ yrs & 44 schools & Illness & Weekly \\
\hline Ma et al [46] & 2015 & Sweden & $\begin{array}{l}\text { Seasonal } \\
\text { influenza }\end{array}$ & Retrospective & $6-16$ yrs. ${ }^{c}$ & 500 schools & Illness & Not specified \\
\hline Mann et al [41] & 2011 & USA & $\begin{array}{l}\text { Pandemic } \\
\text { influenza }\end{array}$ & Prospective & $5-18 \mathrm{yrs}$ & 349 schools & All cause & Daily \\
\hline Mook et al [47] & 2007 & UK & $\begin{array}{l}\text { Seasonal } \\
\text { influenza }\end{array}$ & Prospective & $4-16 y r s$ & 11 schools & Illness & Daily \\
\hline Schmidt et al [48] & 2010 & UK & Seasonal influenza & Retrospective & $5-11$ yrs & 6 schools & Illness & Not specified \\
\hline Suzue et al [50] & 2012 & Japan & $\begin{array}{l}\text { Pandemic } \\
\text { influenza }\end{array}$ & Retrospective & $3-18$ yrs & 142 schools & $\begin{array}{l}\text { Syndrome } \\
\text { specific }\end{array}$ & Daily \\
\hline Williams et al. [42] & 2013 & USA & $\begin{array}{l}\text { Pandemic } \\
\text { influenza }\end{array}$ & Prospective & $5-17 y r s$ & 216 schools & $\begin{array}{l}\text { All cause + } \\
\text { syndrome } \\
\text { specific }\end{array}$ & Weekly \\
\hline
\end{tabular}

\footnotetext{
${ }^{a}$ Medical absences include illness absence and absence to attend medical appointments

${ }^{b}$ Public Health Units (PHUs) varied in size and were broadly divided into large PHUs (population $>400,000$ ) and small PHUs (population $\leq 400,000$ ). Each PHU had a custom surveillance system to measure school absenteeism, collecting data on all cause absenteeism (8 PHUs), illness absenteeism (1 PHU) and respiratory illness absence (1 PHU) from schools within their area

c Not clearly specified. The school ages noted are for compulsory education in the country of study
}

absences $[42,49,50]$. One paper reported medical absences, which combined both illness and planned medical appointments [51]. Another reported data from across multiple health authorities, each of which had a different system in place, varying between all-cause absence, illness absence and respiratory absence [52].

The frequency of data submissions from schools varied between daily [39-41, 47, 49, 50] and weekly reports [21, $42-45,51]$. Weekly reports often contained details of daily absences, so the frequency of reporting did not necessarily affect the level to which the data were analysed. Most studies analysed either daily or weekly absence rates but five studies used exceedances over a threshold as an indicator of a suspected outbreak [41, 42, 45, 49, 52]. One additional study used an absence threshold at city-level to determine the beginning and end points of the H1N1 influenza pandemic [50]. Outbreak definitions varied and are detailed in Table 2.
The majority of studies aggregated absences across geographical areas or groups of schools, with only five studies considering absences at the individual schoollevel [41, 42, 45, 48, 49].

\section{Estimates of the burden of absenteeism}

No standard measure of absenteeism was used across the included studies. Therefore, we were unable to conduct a pooled estimate of the impact of illness or influenza on school absences. Studies reported a mix of baseline absences, peak absences or both, either aggregated across all school age groups or by school type. Six studies reported rates of illness absenteeism (Fig. 3). Baseline illness absences varied from 2.3-3.7\%, [43, 44, $48,50,51]$ with peak illness absence ranging from 4.1 to $9.8 \%[43,44,47,50,51]$. Two studies reported all-cause absenteeism rates, with results varying from $4.4-17.8 \%$ $[39,40]$. The higher reported values were for older 
Table 2 Outbreak definitions used within included studies

\begin{tabular}{lll}
\hline First Author \& year of publication & Outbreak threshold / alert & Time period of breach \\
\hline Chu $2013[52]$ & Exceedance based on C2-MEDIUM method ${ }^{\text {a }}$ & Single day \\
& OR $>5 \%$ all-cause absenteeism ${ }^{b}$ & Single day \\
Kom Mogto $2012[49]$ & $\geq 10 \%$ ILI-related absenteeism & Single day \\
Lenaway $1995[45]$ & $>7.5 \%$ illness absence & Single week average \\
Mann $2011[41]$ & $\geq 8 \%$ all-cause absenteeism AND & Single day \\
Suzue $2012^{C}[50]$ & 1 SD above 30 day mean & Single day \\
Williams $2013[42]$ & $>2 \%$ ILI-related absenteeism & 2 or more consecutive \\
& $>10 \%$ all-cause absenteeism & school days \\
\hline
\end{tabular}

a C2-MEDIUM method calculates the mean and standard deviation (SD) from -9 to -3 days before the day of interest. Threshold is an exceedance of the expected value by three standard deviations

${ }^{b}$ Not clearly stated, assumed from description of methods

c Threshold used to detect start and end of pandemic

children aged 14-18 yrs. Four papers did not directly report on either the percentage of absenteeism or the number of exceedances identified, but instead reported only trends or correlations $[21,42,45,46]$.

Estimates of correlation with health surveillance measures The majority of studies used laboratory isolates as the conclusive marker of influenza activity. Other surveillance measures used for comparison included primary care consultations, hospitalisations or emergency department attendances, telehealth calls and death certifications. The relationship between school absenteeism and established surveillance measures was investigated by study authors using visual inspection and correlation coefficients. The measures of correlation varied and as a result it was not possible to generate a pooled estimate. Tests of correlation included Spearman Rank, Pearson's $r$, cross-correlation analysis and the coefficient of determination.

The correlation between all-cause absenteeism and other surveillance measures were explored in four studies. Whilst visual inspection suggested that peaks in all-cause absenteeism coincided with community outbreaks, [39] correlations between laboratory reports and both all-cause absences and outbreaks based on $>10 \%$ all-cause absence were low $\left(r=0.33^{1}\right.$ and $r=0.39 *$ respectively, $n=216$ schools) [42]. In a study of 80 schools, all-cause absenteeism was not correlated with ILI emergency department visits during periods of low influenza activity $\left(r_{\mathrm{s}}=0.23, p=0.16\right)$, but there was evidence of correlation during periods of high influenza activity $\left(\mathrm{r}_{\mathrm{s}}=0.98, p=0.05\right)$ [40]. A study of outbreak alerts, based on all-cause absenteeism at 349 schools, generated a high number of alerts (one quarter of schools over a 6 week period), only $10 \%$ of which were subsequently confirmed as influenza [41].

\footnotetext{
${ }^{1} P$-value not reported within study paper
}

Two studies explored the correlation between syndrome-specific absenteeism and other surveillance measures. Based on data from over 3400 schools, strong correlations were reported between the number of schools who reported $>10 \%$ ILI-related absence and both laboratory isolates and influenza hospitalisations $\left(\mathrm{r}_{\mathrm{s}}=0.90, p<\right.$ 0.02 and $r_{s}=0.83, p=0.01$ respectively) [49]. Amongst a smaller number of schools $(n=216)$, there was evidence of correlation between the lower threshold of $>5 \%$ ILIrelated absence and laboratory isolates, but the correlation coefficient was reduced $\left(\mathrm{r}=0.78^{*}\right)$ [42]. The highest reported correlations were between laboratory isolates and ILI-absence rates $\left(r=0.92^{*}\right)$, which increased when ILI absences were shifted back by one week $(r=0.97 *)$, suggesting that trends in ILI absences preceded laboratory reports by one week [42].

Studies exploring illness absenteeism presented mixed results. Based on visual inspection, study authors concluded that the peaks of illness absenteeism preceded or were concurrent with peaks in other surveillance systems across influenza seasons [21, 45, 47]. However, correlation with laboratory data varied between no correlation ( $n=373$ schools), [43] mild to moderate correlation $\left(\mathrm{r}=0.11-0.45^{*}\right.$ and cross-correlation $=0.52$, $p<0.001, n=500$ and 6 schools respectively), $[46,48]$ and strong correlation $(\mathrm{r}=0.9, p<0.01, n=187$ schools $)$ [44]. The study of 187 schools also reported correlations with ILI hospitalisations $(\mathrm{r}=0.9, p<0.01)$ and ILIrelated deaths $(\mathrm{r}=0.7, p<0.01)$ [44]. Associations with primary care data ranged from moderate positive correlations to negative correlations $\left(\mathrm{r}=-0.19-0.47,{ }^{2} n=\right.$ 373-500 schools), $[43,46]$ and no association was found with telehealth calls [43]. In a study exploring absences at 27 schools, linear regression modelling identified statistically significant associations between medical absences, which include planned appointments, and both

${ }^{2} P$-value not reported within study paper 


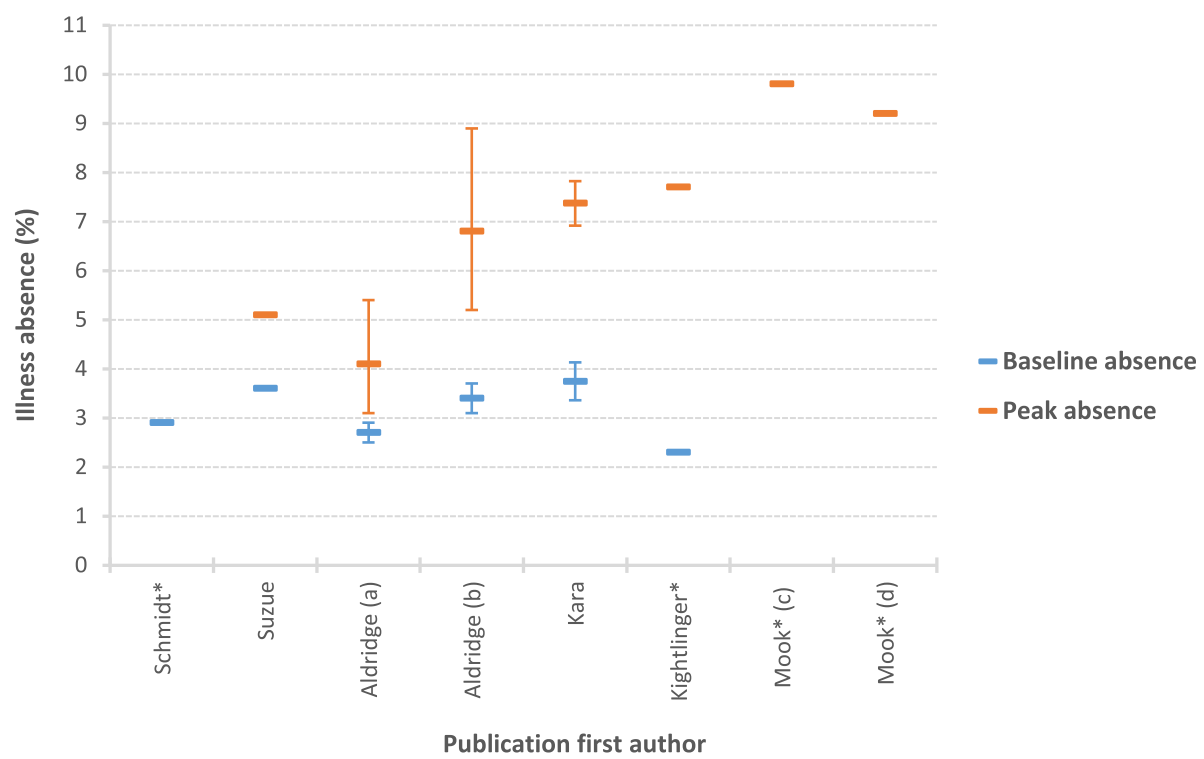

Fig. 3 Percentage of illness absenteeism at baseline and peak during influenza season or outbreak, with 95\% confidence intervals. *No published confidence interval; (A) 2011/12 estimate; (B) 2012/13 estimate; (C) 4-11 year olds; (D) 11-16 year olds

primary care data and laboratory reports $\left(\mathrm{r}^{2}=0.42, p<\right.$ 0.001 and $r^{2}=0.27, p<0.001$ respectively) [51]. The association with primary care ILI reports was strengthened when this surveillance measure was limited to children aged $5-14\left(r^{2}=0.62, p<0.001\right)$.

\section{Lead and lag times}

Thirteen studies considered the lead or lag time of school absence data compared to other surveillance measures. All-cause absenteeism was not found to contribute significantly in terms of timeliness, with the majority of peaks occurring after other surveillance systems, $[39,52]$ and multiple peaks observed which were unrelated to influenza activity [42].

Illness absence presented a mixed picture, with the timeliness of peaks varying between no lead or lag time, [43, 45, 47, 48] a 1-4 week lead time, [21, 45-47, 51] and a lag time of 1-11 weeks [44, 46]. Syndrome-specific absences peaked concurrently or 1-2 weeks before other surveillance measures, [42, 49] and provided lead time on the start, peak and end point of the $\mathrm{H} 1 \mathrm{~N} 1$ pandemic (5 day, 10 day and 17 day lead time respectively) [50].

\section{Discussion}

This systematic review identified fifteen papers which explored the utility of school attendance registers in the syndromic surveillance of infectious disease. All of the papers identified were concerned with influenza, either pandemic or seasonal. There was a particular cluster of papers published following the $2009 \mathrm{H} 1 \mathrm{~N} 1$ pandemic, indicating the heightened need for community-based surveillance systems during the pandemic. None of the papers we identified considered other common infectious diseases, such as diarrhoea and vomiting.

The specificity of the data collected varied between all-cause absenteeism, illness absenteeism and syndrome-specific (in this case ILI) absenteeism. Syndrome-specific absenteeism had the strongest correlation with other surveillance systems, with illness absenteeism generating mixed results and all-cause absenteeism performing the least well. A similar pattern of results emerged in terms of lead and lag times, with ILI-specific absence providing a 1-2 week lead time, compared to lag times reported for all-cause absence data and inconsistent results for illness absence data. These results would indicate a potential role for syndrome-specific absences in the surveillance of influenza. However, all three studies which utilised syndrome-specific absence were conducted during the $\mathrm{H} 1 \mathrm{~N} 1$ pandemic, and therefore the results presented may not reflect the performance of these data in nonpandemic situations. It should also be considered whether a two week lead time is sufficient warning to allow additional protective measures to be put in place.

The three studies which used syndrome-specific data also utilised absence thresholds, which were used to trigger alerts at the individual school level. The thresholds used were $>2,>5 \%$ and $\geq 10 \%$ ILI-related absenteeism. Whilst the $\geq 10 \%$ threshold provided the strongest correlation with other surveillance measures, it provided less lead time than the $>5$ and $>2 \%$ thresholds. The scarcity of papers in this area makes it difficult to explore this further, but such thresholds inevitably result in a trade-off between accuracy and timeliness. Absence 
thresholds may also be influenced by health protection strategies targeted at children, such as vaccination schemes. Such interventions would be expected to reduce peak absence rates and consequently lower thresholds may be required to trigger alerts.

The development of absence thresholds requires an understanding of baseline rates of absence and these have been found to vary by age group. All-cause absenteeism was highest in older children, which could represent absences from causes other than illness. In contrast, both illness absence and symptom-specific absence appeared higher in younger children [43, 47, 49]. There was some indication that influenza started and peaked earlier in younger children, [43, 47] with high schools being affected later [50]. This is consistent with evidence that young children may be the first affected by seasonal and pandemic diseases, [34-36] and highlights the potential value in monitoring infectious illness in elementary/primary school children as an early warning of circulating infections.

As the potential lead time of school absence data was 1-2 weeks, the frequency of data submissions from schools is important in ensuring the early warning is optimised. Whilst the frequency of data reports from schools did not appear to affect correlation with other surveillance systems, the reported 5 day lead time on the start of the H1N1 pandemic may not have provided advanced warning had the data been transferred weekly as oppose to daily. If absence data were utilised to detect and manage outbreaks at the individual school level, daily data submissions would confer additional benefit over weekly reports and aid in the more timely management of localised outbreaks.

\section{Limitations of school absence data}

In the studies identified there was variation in the type of school data used, both between countries and across different health authorities within countries. This makes aggregation of absence data across large areas difficult, [53] and could limit the utility of such data at a national level. School holidays result in a natural break in school attendance data, which is problematic for its use in tracking ongoing community outbreaks. There are also multiple factors which can affect school attendance, making its use in surveillance challenging. All-cause absences will not only capture illness but also unauthorised absences, and has been shown to increase around school holidays [39]. Illness absence will be affected by other infections, such as diarrhoea and vomiting, and has also been found to vary by day of the week [48]. This may contribute to the lack of correlation observed with allcause and illness absence data, especially during periods of low influenza activity. Increases in school absences may also be affected by media coverage of pandemics or high profile deaths in children, $[43,52]$ potentially driven by parental concerns of children catching illnesses at school, or lowering their threshold for keeping a child at home if they are unwell. Whilst this is more likely to be an issue in pandemic influenza, which receives significant media coverage, any high profile outbreak is likely to create the same effect, regardless of the underlying organism.

\section{Conclusion}

The evidence of the utility of school attendance registers in the surveillance of infectious illness in children is limited to studies concerned with influenza. Therefore, the findings of this review may not be applicable to other conditions, such as diarrhoea and vomiting. There is a high level of heterogeneity between studies, making it impractical to pool results and generate a meaningful estimate of either burden of illness absenteeism or its correlation with other surveillance measures. However, the studies identified suggest good correlation between syndrome-specific absences and healthcare surveillance data, with a potential lead time especially from absences in younger school age groups. Further research should consider the utility of school attendance registers for conditions other than influenza, to broaden our understanding of the potential application of this data for infectious disease surveillance in children.

\section{Abbreviations \\ ILI: Influenza-like-illness; OECD: The Organisation for Economic Co-operation and Development; SD: Standard deviation}

\section{Supplementary Information}

The online version contains supplementary material available at https://doi. org/10.1186/s12879-021-06444-6.

Additional file 1. Search terms by database. Details of search terms used by database

Additional file 2. Data extraction form. Standardised form used in the data extraction process

\section{Acknowledgements}

Not applicable.

\section{Authors' contributions}

$A D, J L H, J P H, R V$ and $S O B$ all contributed to the study design. AD and JLH undertook the search and article screening. AD and JPH undertook the full text review, data extraction and analysis. AD wrote the first draft. All authors read and approved the final manuscript.

\section{Funding}

This research was funded by the National Institute for Health Research Health Protection Research Unit (NIHR HPRU) in Gastrointestinal Infections at University of Liverpool in partnership with Public Health England (PHE), in collaboration with University of East Anglia, University of Oxford and the Quadram Institute [Grant number NIHR HPRU 2012-10038]. The funding body had no role in the design of this study, nor in the collection, analysis, and interpretation of data and writing of the manuscript. The views expressed are those of the authors and not necessarily those of the NHS, the NIHR, the Department of Health and Social Care or Public Health England. 


\section{Availability of data and materials}

The datasets used and/or analysed during the current study are available from the corresponding author on reasonable request.

\section{Declarations}

Ethics approval and consent to participate

Not applicable.

\section{Consent for publication}

Not applicable.

\section{Competing interests}

The authors declare that they have no competing interests.

\section{Author details}

${ }^{1} \mathrm{NIHR}$ Health Protection Research Unit in Gastrointestinal Infections, University of Liverpool, Liverpool, UK. ${ }^{2}$ Institute of Population Health Sciences, University of Liverpool, Liverpool, UK. ${ }^{3}$ Field Epidemiology Service, Public Health England, Liverpool, UK.

Received: 10 December 2019 Accepted: 20 July 2021

Published online: 03 August 2021

\section{References}

1. World Health Organization. Public health surveillance. WHO. 2017. https://www.who.int/topics/public_health_surveillance/en/. Accessed 1 Oct 2018.

2. Jajosky RA, Groseclose SL. Evaluation of reporting timeliness of public health surveillance systems for infectious diseases. BMC Public Health. 2004;4(1):29. https://doi.org/10.1186/1471-2458-4-29.

3. Morse SS. Public health surveillance and infectious disease detection. Biosecurity Bioterrorism Biodefense Strateg Pract Sci. 2012;10(1):6-16. https://doi.org/10.1089/bsp.2011.0088.

4. Henning KJ. What is syndromic surveillance? MMWR. 2004;53:7-11.

5. Elliot A. Syndromic surveillance: the next phase of public health monitoring during the H1N1 influenza pandemic? Euro Surveill. 2009;14:19391. https:// doi.org/10.2807/ese.14.44.19391-en

6. Heffernan R, Mostashari F, Das D, Karpati A, Kulldorff M, Weiss D. Syndromic surveillance in public health practice, New York City. Emerg Infect Dis. 2004; 10(5):858-64. https://doi.org/10.3201/eid1005.030646.

7. Hope K, Durrheim DN, d'Espaignet ET, Dalton C. Syndromic surveillance: is it a useful tool for local outbreak detection? J Epidemiol Community Health. 2006;60(5):374-5. https://doi.org/10.1136/jech.2005.035337.

8. Katz R, May L, Baker J, Test E. Redefining syndromic surveillance. J Epidemiol Glob Health. 2011;1(1):21-31. https://doi.org/10.1016/J.JEGH.2011.06.003.

9. Elliot AJ, Hughes HE, Hughes TC, Locker TE, Shannon T, Heyworth J, et al. Establishing an emergency department syndromic surveillance system to support the London 2012 Olympic and Paralympic games. Emerg Med J. 2012;29(12):954-60. https://doi.org/10.1136/emermed-2011-200684.

10. Loveridge P, Cooper D, Elliot AJ, Harris J, Gray J, Large S, et al. Vomiting calls to NHS direct provide an early warning of norovirus outbreaks in hospitals. J Hosp Infect. 2010;74(4):385-93. https://doi.org/10.1016/j.jhin.2009.10.007.

11. Heffernan R, Mostashari F, Das D, Besculides M, Rodriguez C, Greenko J, et al. New York City syndromic surveillance systems. MMWR. 2004; 53(Suppl):25-7.

12. Bork KH, Klein BM, Mølbak K, Trautner S, Pedersen UB, Heegaard E. Surveillance of ambulance dispatch data as a tool for early warning. Eurosurveillance. 2006;11(12):13-4. https://doi.org/10.2807/esm.11.12. 00669-en

13. Moore K. Real-time syndrome surveillance in Ontario, Canada: the potential use of emergency departments and telehealth. Eur J Emerg Med. 2004; 11(1):3-11. https://doi.org/10.1097/00063110-200402000-00002.

14. Blozik E, Grandchamp C, Von Overbeck J. Influenza surveillance using data from a telemedicine centre. Int J Public Health. 2012;57(2):447-52. https:// doi.org/10.1007/s00038-011-0240-1.

15. Ginsberg J, Mohebbi MH, Patel RS, Brammer L, Smolinski MS, Brilliant L. Detecting influenza epidemics using search engine query data. Nature. 2009;457(7232):1012-4. https://doi.org/10.1038/nature07634.
16. Milinovich GJ, Williams GM, Clements ACA, Hu W. Internet-based surveillance systems for monitoring emerging infectious diseases. Lancet Infect Dis. 2014;14(2):160-8. https://doi.org/10.1016/S1473-3099(13)70244-5.

17. Polgreen PM, Chen Y, Pennock DM, Nelson FD, Weinstein RA. Using internet searches for influenza surveillance. Clin Infect Dis. 2008;47(11):1443-8. https://doi.org/10.1086/593098.

18. Elliot AJ, Kara EO, Loveridge P, Bawa Z, Morbey RA, Moth M, et al. Internetbased remote health self-checker symptom data as an adjuvant to a national syndromic surveillance system. Epidemiol Infect. 2015;143(16):341622. https://doi.org/10.1017/S0950268815000503.

19. Hulth A, Rydevik $G$, Linde A. Web queries as a source for syndromic surveillance. PLoS One. 2009;4(2):e4378. https://doi.org/10.1371/journal.pone. 0004378.

20. Edge VL, Pollari F, Lim G, Aramini J, Sockett P, Martin SW, et al. Syndromic surveillance of gastrointestinal illness using pharmacy over-the-counter sales. Can J Public Heal. 2004;95(6):446-50. https://doi.org/10.1007/BF03403991.

21. Bollaerts K, Antoine J, Robesyn E, van Proeyen L, Vomberg J, Feys E, et al. Timeliness of syndromic influenza surveillance through work and school absenteeism. Arch Public Heal. 2010;68:115-20. https://doi.org/10.1186/ 0778-7367-68-3-115.

22. Zhao H, Joseph CA, Phin N. Outbreaks of influenza and influenza-like illness in schools in England and Wales, 2005/06. Eurosurveillance. 2007;12(5):3-4. https://doi.org/10.2807/esm.12.05.00705-en.

23. Azor-Martinez E, Gonzalez-Jimenez Y, Luisa Seijas-Vazquez M, CobosCarrascosa E, Santisteban-Martinez J, Miguel Martinez-Lopez J, et al. The impact of common infections on school absenteeism during an academic year. Am J Infect Control. 2014;42:632-7. https://doi.org/10.1016/j.ajic.2014. 02.017 .

24. Sandora TJ, Shih MC, Goldmann DA. Reducing absenteeism from gastrointestinal and respiratory illness in elementary school students: A randomized, controlled trial of an infection-control intervention. Pediatrics. 2008;121:e1555-62. https://doi.org/10.1542/peds.2007-2597.

25. White CG, Shinder FS, Shinder AL, Dyer DL. Reduction of illness absenteeism in elementary schools using an alcohol-free instant hand sanitizer. J Sch Nurs. 2001;17(5):248-65. https://doi.org/10.1177/10598405010170050401.

26. Nandrup-Bus I. Mandatory handwashing in elementary schools reduces absenteeism due to infectious illness among pupils: A pilot intervention study. Am J Infect Control. 2009;37:820-6. https://doi.org/10.1016/j.ajic.2009. 06.012 .

27. Viboud C, Boëlle P-Y, Cauchemez S, Lavenu A, Valleron A-J, Flahault A, et al. Risk factors of influenza transmission in households. Br J Gen Pract. 2004; 54(506):684-9.

28. Principi N, Esposito S, Gasparini R, Marchisio P, Crovari P, Flu-Flu SG. Burden of influenza in healthy children and their households. Arch Dis Child. 2004; 89(11):1002-7. https://doi.org/10.1136/adc.2003.045401.

29. Barker J, Stevens D, Bloomfield SF. Spread and prevention of some common viral infections in community facilities and domestic homes. J Appl Microbiol. 2001;91(1):7-21. https://doi.org/10.1046/j.1365-2672.2001.01364.x.

30. Neuzil KM, Hohlbein C, Zhu YW. Illness among schoolchildren during influenza season - effect on school absenteeism, parental absenteeism from work, and secondary illness in families. Arch Pediatr Adolesc Med. 2002; 156(10):986-91. https://doi.org/10.1001/archpedi.156.10.986.

31. Glass LM, Glass RJ. Social contact networks for the spread of pandemic influenza in children and teenagers. BMC Public Health. 2008;8(1):61. https:// doi.org/10.1186/1471-2458-8-61.

32. Béraud G, Kazmercziak S, Beutels P, Levy-Bruhl D, Lenne X, Mielcarek N, et al The French connection: the first Large population-based contact survey in France relevant for the spread of infectious diseases. PLoS One. 2015;10(7): e0133203. https://doi.org/10.1371/journal.pone.0133203.

33. Cauchemez S, Bhattarai A, Marchbanks TL, Fagan RP, Ostroff S, Ferguson NM, et al. Role of social networks in shaping disease transmission during a community outbreak of 2009 H1N1 pandemic influenza. Proc Natl Acad Sci U S A. 2011;108(7):2825-30. https://doi.org/10.1073/pnas.1008895108.

34. Cate TR. Clinical manifestations and consequences of influenza. Am J Med. 1987;82(6 SUPPL. 1):15-9.

35. Glezen WP, Couch RB. Inter pandemic influenza in the Houston area 19741976. N Engl J Med. 1978;298(11):587-92. https://doi.org/10.1056/NEJM1 97803162981103

36. Cox NJ, Subbarao K. Global epidemiology of influenza: past and present. Annu Rev Med. 2000;51(1):407-21. https://doi.org/10.1146/annurev.med. 51.1.407. 
37. Donaldson A, Hardstaff J, Harris J, Vivancos R, O'Brien S. School-based surveillance of acute infectious disease in children: a systematic review. PROSPERO. 2019:CRD42019119737. https:/www.crd.york.ac.uk/prospero/ display_record.php?ID=CRD42019119737.

38. Oecd Countries. 2019. http://worldpopulationreview.com/countries/oecdcountries/.

39. Besculides M, Heffernan R, Mostashari F, Weiss D. Evaluation of school absenteeism data for early outbreak detection, New York City. BMC Public Health. 2005:5:105.

40. Crawford GB, McKelvey S, Crooks J, Siska K, Russo K, Chan J. Influenza and school-based influenza-like illness surveillance: a pilot initiative in Maryland. Public Health Rep. 2011;126:591-6.

41. Mann P, O'Connell E, Zhang G, Llau A, Rico E, Leguen FC. Alert system to detect possible school-based outbreaks of influenza-like illness. Emerg Infect Dis. 2011:17(2):262-4. https://doi.org/10.3201/eid1702.100496.

42. Williams NJ, Ghosh TS, Bisgard KM, Vogt RL. Comparison of 3 schoolbased influenza surveillance indicators: lessons learned from 2009 pandemic influenza a (H1N1)-Denver metropolitan region, Colorado. J Public Heal Manag Pract. 2013;19(2):119-25. https://doi.org/10.1097/PHH. Ob013e318252fo05.

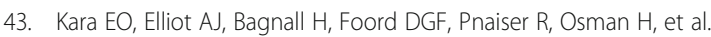
Absenteeism in schools during the 2009 influenza a(H1N1) pandemic: a useful tool for early detection of influenza activity in the community? Epidemiol Infect. 2012;140(7):1328-36. https://doi.org/10.1017/50950268811 002093.

44. Kightlinger L, Horan V. School illness absenteeism during 2009 influenza a (H1N1) pandemic--South Dakota, 2009-2010. S D Med. 2013;66(177):179-81.

45. Lenaway DD, Ambler A. Evaluation of a school-based influenza surveillance system. Public Health Rep. 1995;110(3):333-7.

46. Ma T, Englund H, Bjelkmar P, Wallensten A, Hulth A. Syndromic surveillance of influenza activity in Sweden: an evaluation of three tools. Epidemiol Infect. 2015;143(11):2390-8. https://doi.org/10.1017/S0950268814003240.

47. Mook P, Joseph C, Gates P, Phin N. Pilot scheme for monitoring sickness absence in schools during the 2006/07 winter in England: can these data be used as a proxy for influenza activity? Euro Surveill. 2007;12(12):E11-2. https://doi.org/10.2807/esm.12.12.00755-en.

48. Schmidt WP, Pebody R, Mangtani P. School absence data for influenza surveillance: A pilot study in the United Kingdom. Euro Surveill. 2010;15: 19467. https://doi.org/10.2807/ese.15.03.19467-en

49. Kom Mogto CA, De Serres G, Douville Fradet M, Lebel G, Toutant S, Gilca R, et al. School absenteeism as an adjunct surveillance Indicator: experience during the second wave of the 2009 H1N1 pandemic in Quebec, Canada. PLoS One. 2012;7(3):e34084. https://doi.org/10.1371/journal.pone.0034084.

50. Suzue T, Hoshikawa Y, Nishihara S, Fujikawa A, Miyatake N, Sakano N, et al. The New School Absentees Reporting System for Pandemic Influenza AV H1N1 2009 Infection in Japan. PLoS One. 2012;7:e30639.

51. Aldridge RW, Hayward AC, Field N, Warren-Gash C, Smith C, Pebody R, et al. Are school absences correlated with influenza surveillance data in England? Results from decipher my data-a research project conducted through scientific engagement with schools. PLoS One. 2016;11(3):e0146964. https:// doi.org/10.1371/journal.pone.0146964.

52. Chu A, Savage R, Whelan M, Rosella LC, Crowcroft NS, Willison D, et al. Assessing the Relative Timeliness of Ontario's Syndromic Surveillance Systems for Early Detection of the 2009 Influenza H1N1 Pandemic Waves. Can J Public Heal. 2013;104:e340-7. https://doi.org/10.17269/cjph.104.3721.

53. Enanoria WTA, Crawley AW, Tseng W, Furnish J, Balido J, Aragon TJ. The epidemiology and surveillance response to pandemic influenza a (H1N1) among local health departments in the san Francisco bay Area. BMC Public Health. 2013;13:276. https://doi.org/10.1186/1471-2458-13-276.

\section{Publisher's Note}

Springer Nature remains neutral with regard to jurisdictional claims in published maps and institutional affiliations.

Ready to submit your research? Choose BMC and benefit from:

- fast, convenient online submission

- thorough peer review by experienced researchers in your field

- rapid publication on acceptance

- support for research data, including large and complex data types

- gold Open Access which fosters wider collaboration and increased citations

- maximum visibility for your research: over $100 \mathrm{M}$ website views per year

At BMC, research is always in progress.

Learn more biomedcentral.com/submissions 